EPJ Web of Conferences 73, 08006 (2014)

DOI: $10.1051 /$ epjconf/20147308006

(C) Owned by the authors, published by EDP Sciences, 2014

\title{
The Italian Tau/charm project
}

\author{
Maria Enrica Biagini ${ }^{\text {a }}$ \\ INFN Frascati National Laboratories, Accelerator Division, via E. Fermi 40, 00044 Frascati, Italy
}

\begin{abstract}
A $\tau /$ charm Factory, an e + e- collider with very high luminosity at the $2-4.6 \mathrm{GeV}$ center of mass energy, to be built on the Rome University at Tor Vergata campus, was studied by the Consortium Nicola Cabibbo Laboratory and the INFN Frascati Laboratories. This project is the natural evolution of the flagship Italian project SuperB Factory, funded by the Italian Government in 2010 with a budget that turned out to be insufficient to cover the total costs of the project. The study of rare events at the $\tau /$ charm energy was already planned as a Phase-II of SuperB [1]. This design keeps all the unique features of SuperB, including the polarization of the electron beam, with the possibility to take data in a larger energy range, with reduced accelerator dimensions and construction and operation costs. A Report on the accelerator design has been published in September 2013 [2].
\end{abstract}

\section{Introduction}

The project of a $\tau /$ charm Factory in the Rome Tor Vergata University area was developed after the cancellation of the SuperB B-Factory project. The principles of operation of such an accelerator are still based on the SuperB ones, like the "Crab waist and large Piwinski angle" [3] collision scheme, which has been successfully tested at the $\Phi$-Factory DAФNE in Frascati [4], with small beam emittances and smaller beam sized at the Interaction Point. As a plus the $\tau /$ charm, due to the lower beam energy, will have very low power consumption and running costs.

The accelerator is designed to have a main operation point at the $\tau /$ charm threshold, however operation at lower and slightly higher energies is also foreseen. The lower centre of mass energy should be at the $\Phi$ resonance $(1.05 \mathrm{GeV})$ to complete the data collected at the DAФNE collider at LNF Frascati. Also $2 \mathrm{GeV}$ in the centre of mass will provide interesting data at the threshold of the nucleon antinucleon production, for studies of the nucleon form factors. Upper energies will allow the study of the $\Lambda$ s resonance at $4.35 \mathrm{GeV}$. For this reason the maximum energy is $2.3 \mathrm{GeV}$.

The new design is based on a symmetric beams collision, rather than the asymmetric one planned for SuperB. This is justified by the different processes, which will be studied, and makes the design a lot simpler. For example, for SuperB a large effort was put on the design of the Final Focus (FF) sections, where the two beams are brought into collision, especially on a state-of-the-art design of the first superconducting quadrupole doublet, able to cope with the high gradients required and the small space available. For the $\tau /$ charm these elements will have more relaxed characteristics, and the

\footnotetext{
a e-mail: marica.biagini@lnf .infn.it
}

This is an Open Access article distributed under the terms of the Creative Commons Attribution License 4.0, which permits unrestricted use, distribution, and reproduction in any medium, provided the original work is properly cited. 
symmetric FF will easy the design. The beam parameters will be similar to those planned for SuperB, yet more relaxed due to the lower beam energy. The experience with SuperB, for which an extensive study on collective effects has been carried out, turns out now to be very useful in the choice on which are the most critical parameters. The electron beam polarization, a unique feature, will also be part of this design. However, as a consequence of the lower energy and the larger spin depolarization time, the design of the "spin rotation system" (SRS) will be a lot simpler. Instead of two SRS, one on each side of the FF, just one, with similar characteristics, will be placed in the straight section opposite to the IP. This insertion is called "Siberian Snake". Thanks to this choice, there are no more polarization resonances, and the option to have asymmetric beam energies would not require to have "quantized" energies.

The use of the Linac for a SASE-FEL facility, a Beam Test Facility for detectors test, and a Neutron production facility have also been considered as options.

\section{Collider beam parameters}

Two Main Rings are the principal component of the collider: electrons and positrons will circulate in two separate rings, crossing at the Interaction Point (IP) where the events will be collected by the Detector. The two Rings, about $350 \mathrm{~m}$ long each, have similar magnetic structure, the only difference being the presence in the electron ring of a Siberian Snake (SS) used to rotate the transverse spin of injected electrons into a longitudinal spin at the IP.

The collision scheme adopted is the "Large Piwinski Angle and Crab Waist" scheme already tested at DAФNE and baseline of the design of the upgraded SuperKEKB B-Factory at KEK in Japan. The characteristics of this scheme are the large crossing angle, which reduces the parasitic crossings and the beam overlap area useful for the luminosity, the extremely low beam emittances and IP beam sizes, and the use of sextupoles to cancel the resonances appearing with the crossing angle.

The beam parameters have been chosen in order to have a peak luminosity of $10^{35} \mathrm{~cm}^{-2} \mathrm{sec}^{-1}$ at the $\tau /$ charm threshold and upper. At lower centre of mass energies a lower luminosity, but still an order of magnitude higher than that of present colliders operating in the same energy range, can be achieved with a suitable choice of parameters. In Table 1 is the list of beam parameters relevant for the luminosity, for the energy of $2 \mathrm{GeV} /$ beam. It has to be noted that the emittance, bunch length and energy spread of such intense bunches are dominated (and increased) at these energies by the Intra Beam Scattering (IBS) mechanism. For this reason the numbers in Table 1 include an estimation of this effect, as well as the hourglass effect, which reduces the luminosity due to the bunch length longer than the IP $\beta_{y}$. For the $\tau$ /charm design beam-beam tune shifts are on the safe side: the horizontal is negligible, due to the features of the LPA scheme, the vertical $\xi_{y}$ is about 0.09 for the baseline parameters, a value lower than those routinely achieved at the B-Factories PEP-II and KEKB.

In Fig. 1 is a sketch of the $\tau$ /charm complex in the Tor Vergata University site that was reserved for SuperB.

Another feature of the project is the possibility to collect data at different centre of mass energies, ranging from 2 to $4.6 \mathrm{GeV}$. In Table 2 are listed the main parameters, depending on the beam energy, missing parameters are equal to those in Table 1. At low energy (last column) the insertion of 8 wigglers is foreseen to keep the same damping times. The electron beam polarization will be maximum around $4 \mathrm{GeV}$ c. of $\mathrm{m}$.

\section{Final Focus design}

A Final Focus system is designed to bring the two beams in collision in the Interaction Point (IP) with the design $\beta$-functions. Due to the strong gradients of the IP quadrupoles and to the very low IP $\beta$-functions, the chromaticity of this system needs a complicate sextupole correction scheme, in order to avoid non-linear effects, which can degrade the luminosity. This Interaction Region (IR) is usually 
Table 1. $\tau /$ charm beam parameters.

\begin{tabular}{|l|l|l|}
\hline Parameter & Units & \\
\hline Luminosity & $\mathrm{cm}^{-2} \mathrm{sec}^{-1}$ & $10^{35}$ \\
\hline Beam energy & $\mathrm{GeV}$ & 1 to 2.3 \\
\hline Circumference & $\mathrm{m}$ & 341 \\
\hline Crossing angle & $\mathrm{mrad}$ & 60 \\
\hline$\beta_{x}$ at IP & $\mathrm{cm}$ & 7 \\
\hline$\beta_{y}$ at IP & $\mathrm{cm}$ & 0.06 \\
\hline Horizontal emittance & $\mathrm{nm}$ & 4.9 \\
\hline Vertical emittance & $\mathrm{pm}$ & 12 \\
\hline Bunch length & $\mathrm{mm}$ & 6.9 \\
\hline Horizontal bb tune shift & & 0.004 \\
\hline Vertical bb tune shift & & 0.09 \\
\hline Number of bunches & & 530 \\
\hline Max Beam current & $\mathrm{mA}$ & 1750 \\
\hline
\end{tabular}

Table 2. $\tau /$ charm beam parameters at different energies.

\begin{tabular}{|l|l|l|l|l|}
\hline Parameter & Units & & & \\
\hline Luminosity & $\mathrm{cm}^{-2} \mathrm{sec}^{-1}$ & $10^{35}$ & $10^{35}$ & $10^{34}$ \\
\hline Beam energy & $\mathrm{GeV}$ & 2.3 & 2.0 & 1.0 \\
\hline Horizontal emittance & $\mathrm{nm}$ & 4.9 & 5.1 & 6.1 \\
\hline Vertical emittance & $\mathrm{pm}$ & 12 & 13 & 15 \\
\hline Bunch length & $\mathrm{mm}$ & 6.9 & 6.9 & 10.1 \\
\hline Horizontal bb tune shift & & 0.004 & 0.004 & 0.002 \\
\hline Vertical bb tune shift & & 0.08 & 0.09 & 0.064 \\
\hline Beam currents & $\mathrm{mA}$ & 1720 & 1750 & 1000 \\
\hline Beam power & $\mathrm{MW}$ & 0.28 & 0.16 & 0.05 \\
\hline
\end{tabular}

the most difficult part of the collider to be laid out. The two rings are closely spaced, both the radial and vertical $\beta$-functions reach their maxima and the quadrupoles are the strongest of the lattice, moreover the constraints posed by the detector must be fulfilled too. Permanent magnets (PM) are an attractive technology to solve the final doublet design problems. Its main advantages are compactness, stability, field quality and simplicity. A special design of the IR was carried out in order to maximize the detector solid angle while keeping enough beam pipe aperture to have good lifetime and to avoid beam pipe heating from background particles. Figure 2 shows a cartoon of the near IP region horizontal cross section. The colour boxes represent the PM material (QD0s in green, QD0 in blue, QF1 in red). The 40 $\sigma$ beam stay clears are represented with dashed lines. The thin beam pipe facing the IP is represented with thin red lines. Note the different scale of the radial and longitudinal axes. The two QD0 quadrupoles are shared among the electron and positron machine. 


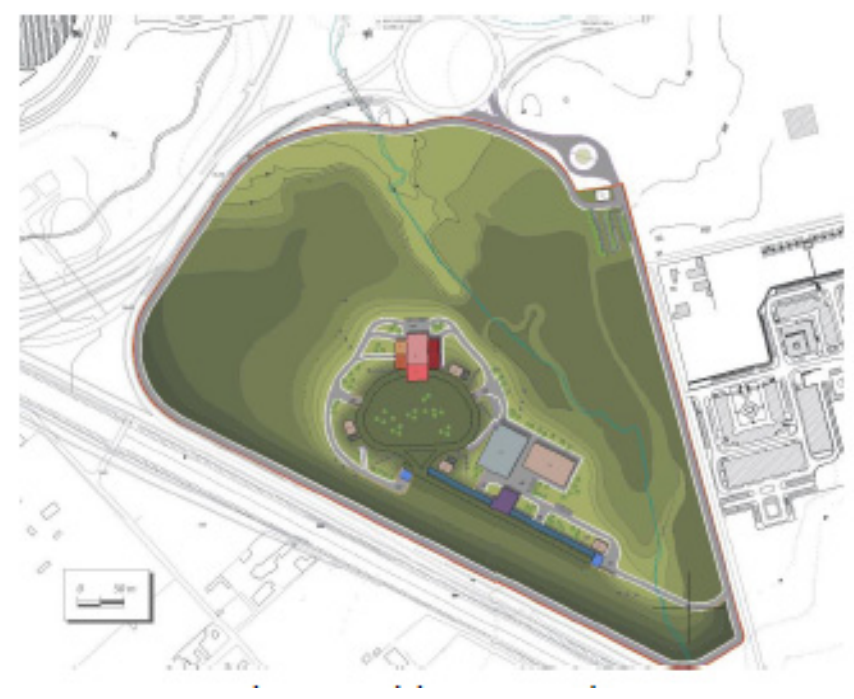

Figure 1. The $\tau /$ charm complex at Tor Vergata.

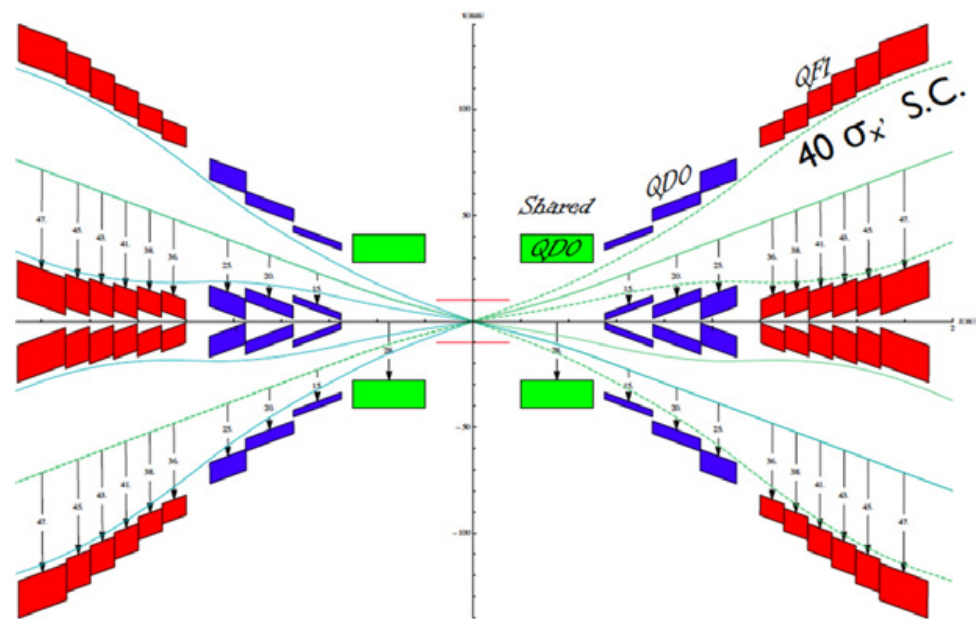

Figure 2. The near IP region horizontal cross section. In green the first defocusing quadrupole, common to both beams.

\section{Injection system}

The layout of the injection system is based on the design of the SuperB injection system (with 3 Linacs). The same design for the linac and Damping Ring (DR) structure is used. The main difference with respect to the SuperB design is the fact that only positrons are stored in the DR (60 m circumference). The system is designed to sustain the stress of a continuous (trickle) injection into the Main Rings $(50 \mathrm{~Hz}, 5$ bunches at a time). As for the SuperB case, the Linacs can be used to accelerate electron pulses for a SASE FEL facility (timing of injection allows for $30 \mathrm{~Hz}$ parasitic operation for FEL). A sketch of the injection system is in Fig. 3. The total electron energy is $2.9 \mathrm{GeV}$, while positrons 


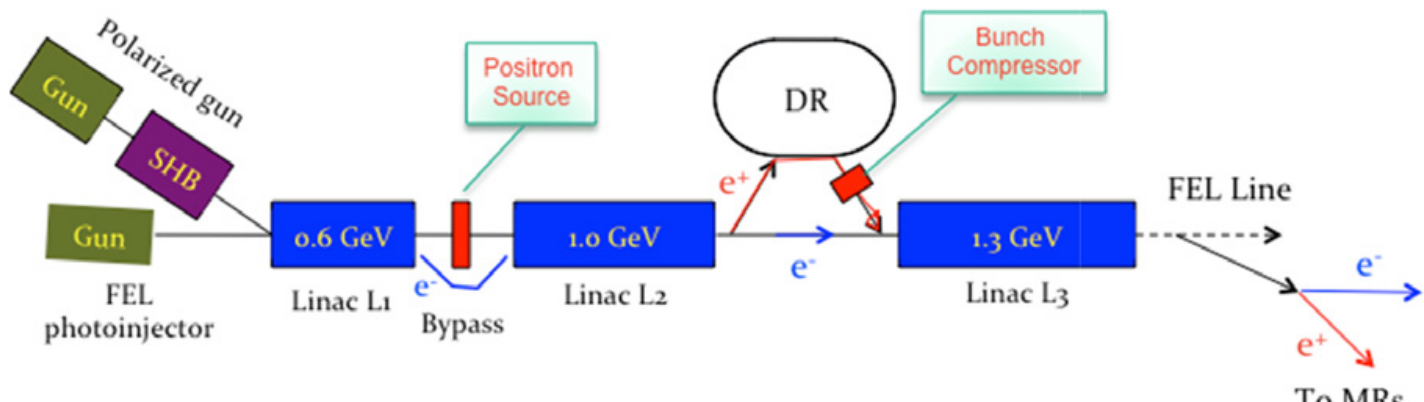

Figure 3. The $\tau /$ charm injection complex.

will reach $2.3 \mathrm{GeV}$. The number of klystrons and cavities in the design allows reaching the maximum positron energy of $2.3 \mathrm{GeV}$ also with one klystron off.

The possibility to drive a SASE X-ray FEL using the $2.4 \mathrm{GeV} \tau /$ charm Linac has been studied. To achieve an energy of $6 \mathrm{GeV}$ (for 1.5 and 3 Angstrom photon wavelength) additional Linac sections can be installed at the end of the last Linac, using the C-band ( $\mathrm{f}=5712 \mathrm{MHz}$ ) technology, which is being developed at LNF in the framework of the EU-TIARA project, and will be soon installed at SPARCLAB facility. Assuming an accelerating gradient of $40 \mathrm{MV} / \mathrm{m}$, additional $80 \mathrm{~m}$ of Linac sections (about 40) should be added (total Linac length $300 \mathrm{~m}$ ).

\section{Conclusions}

A new infrastructure for a low energy Flavour Factory, with possible applications in other fields such as FEL and BTF has been designed. A Report on the accelerator design (150 pp.) has been published and can be the base for a fast TDR phase. The Physics case Report is in preparation. The estimated cost of the facility would be entirely covered by the promised "SuperB" funding. However a decision on the future of the Italian Flagship projects has not been taken yet, and the PNR (National Research Plan) for the next 3 years is still under study. The Nicola Cabibbo Laboratory, a Consortium between INFN and Tor Vergata University, is in place to construct and run in case such a facility should be funded.

\section{References}

[1] M.E. Biagini, P. Raimondi, J. Seeman (editors) et al., "SuperB Progress Report: Accelerator", arXiv:1009.6178v3 (2010)

[2] M.E. Biagini (editor) et al., " $\tau /$ charm factory Accelerator Report”, arXiv: 1310.6944 (2013)

[3] P. Raimondi, D. Shatilov, M. Zobov, "Beam-Beam Issues for Colliding Schemes with Large Piwinski Angle and Crabbed Waist”, LNF-07-003-IR, e-Print:physics/0702033 (2007)

[4] M. Zobov et al., "Test of "crab-waist" collisions at DAФNE Ф-Factory", Phys. Rev. Lett. 104, 174801 (2010) 